\title{
Positional, Reorientational and Bond Orientational Order in DNA Mesophases
}

\author{
V. Lorman ${ }^{1}$, R. Podgornik ${ }^{2,3,4}$ and B. Žekšs 5,3 \\ ${ }^{1}$ Laboratoire de Physique Mathematique et Theorique, Universite Montpellier II, F-34095 Montpellier, France \\ 2 Department of Physics, Faculty of Mathematics and Physics, University of Ljubljana, SI-1000 Ljubljana, Slovenia \\ ${ }^{3}$ Department of Theoretical Physics, J.Stefan Institute, SI-1000 Ljubljana, Slovenia \\ ${ }^{4}$ LPSB/NICHHD, Bld. 12A Rm. 2041, National Institutes of Health, Bethesda, MD 20892-5626 \\ ${ }^{5}$ Institute of Biophysics, Medical Faculty, University of Ljubljana, SI-1000 Ljubljana, Slovenia
}

\begin{abstract}
We investigate the orientational order of transverse polarization vectors of long, stiff polymer molecules and their coupling to bond orientational and positional order in high density mesophases. Homogeneous ordering of transverse polarization vector promotes distortions in the hexatic phase, whereas inhomogeneous ordering precipitates crystalization of the $2 \mathrm{D}$ sections with different orientations of the transverse polarization vector on each molecule in the unit cell. We propose possible scenarios for going from the hexatic phase, through the distorted hexatic phase to the crystalline phase with an orthorhombic unit cell observed experimentally for the case of DNA.
\end{abstract}

The microscopic structure of high density DNA mesophases is not only of utmost importance for polymer science in general [1] but has a fundamental bearing also on topics as far removed from one another as the nature of the phase diagram of magnetic vortex arrays in type-II superconductors [2] or the structure of genosomes used for DNA transfection in gene therapy related studies [3]. By virtue of conventional wisdom at high densities DNA should form crystals of hexagonal symmetry in the plane perpendicular to the long axes of the molecules, with long range positional order, and nematic (or better cholesteric) arrays with short range positional order at densities intermediate between the crystalline and isotropic phases. There are however a few refinements one should add to this picture.

First of all it was found experimentally that DNA in fact forms several crystalline phases [4.5]. At very high densities $\mathrm{Li}^{+}$-DNA makes a crystalline phase with orthorhombic symmetry that implies a distorted hexagonal unit cell perpendicular to the long axes. The probable reason for this is that there are angular frustrations between neighboring DNA molecules that make hexagonal local symmetry energetically costly. A similar situation can also be encountered in frustrated spin systems, such as in antiferromagnets on a triangular lattice or alkylchain systems [6]. In all these cases distorting the hexagonal equilateral into isosceles triangles could lower the angular part in the interaction energy. In this way two pairs of molecules are closer to each other, maintaining optimal angles, while the third pair is further apart and can be in a non-optimal configuration.

In addition just below the crystalline phase DNA forms a line hexatic phase with short range positional order and long range bond orientational order [7]. The variation of the positional correlation length as a function of DNA density shows that positional order within this phase is more liquid-like (shorter correlation length) the more DNA density is increased [8]. This trend is surprising and counterintuitive: one would expect the DNA array to exhibit increasingly longer ranged positional order approaching the crystalline phase where it becomes (ideally) infinite. Again the progressive disordering of DNA could be due [8] to increasing angular frustration of molecules as they try to satisfy both the positional and the angular constraints imposed by the interaction potential.

The question of exactly how angular frustrations could affect crystalline and bond-orientational order in DNA arrays will be addressed in this contribution. Angular frustrations of course correspond to angle dependent terms in the interaction free energy between DNAs. Recent theoretical investigations 9] have indeed made it clear that at small enough interaxial separations the interaction free energy depends crucially on the mutual orientation of the two interacting molecules. This orientation can be specified in any $2 \mathrm{D}$ plane perpendicular to the long axes, by giving e.g. the position of the major groove of one molecule with respect to the line joining a pair of them and of the major groove of the other one with respect to the first one [9]. This amounts to effectively defining a $2 \mathrm{D}$ vector associated with each of the interacting molecules (we call it transverse polarization vector, $\mathbf{p}$ ), perpendicular to their long axes, and the interaction between them will depend on their separation as well as their orientation described by their transverse polarization vectors. Basing our hypothesis loosely on [9] we assume that at low DNA density the interaction does not depend on the mutual orientation of molecules, at intermediate densities the interaction is minimal for parallel $\mathbf{p}$ orientation while it is minimal for some finite angle between ps for larger densities.

We assume that the molecules are stiff enough so that ordering of all 2D sections perpendicular to their long axes are the same. We consider consecutively two possible situations. First at intermediate densities the preferred angle between transverse polarization vectors in all $2 \mathrm{D}$ sections is assumed to be zero. The transverse polarization order is thus homogeneous and we describe it with a $2 \mathrm{D}$ constant vector $\mathbf{p}$, in the standard complex form $\mathbf{p}=|p| e^{i \phi}$, where $\phi$ is the local angle between the transverse polarization vector and some preferred axis. In the isotropic $2 \mathrm{D}$ liquid, hexatic order is 
associated with two symmetric combinations of components of a 6 th rank tensor. In the complex form the hexatic order parameter can be written in terms of the angle $\theta$ between molecular bonds in the form $\psi=|\psi| e^{i 6 \theta}$, where $0 \leq \theta \leq \frac{2 \pi}{6}$. The free energy of hexatic ordering now depends only on $\mathcal{F}_{\text {hex }}=\mathcal{F}_{\text {hex }}\left(|\psi|^{2}\right)$, while the free energy of polar correlations can be written analogously as $\mathcal{F}_{\text {pol }}=\mathcal{F}_{\text {pol }}\left(|p|^{2}\right)$. The coupling of these two types of order can be introduced through an interaction term $\mathcal{F}_{\text {int }}=\mathcal{F}_{\text {int }}\left(|\psi||p|^{6} \cos 6(\phi-\theta)\right)$. In this picture the free energy of the isotropic liquid can become unstable with respect to hexatic as well as polar order parameters.

The total free energy $\mathcal{F}=\mathcal{F}_{\text {hex }}+\mathcal{F}_{\text {pol }}+\mathcal{F}_{\text {int }}$ can now be written as

$$
\begin{aligned}
\mathcal{F} & =a_{1}|\psi|^{2}+b_{1}|\psi|^{4}+a_{2}|p|^{2}+b_{2}|p|^{4}+ \\
& +c_{1}|\psi||p|^{6} \cos 6(\phi-\theta)+\ldots
\end{aligned}
$$

This free energy has five different stable solutions as obtained by standard methods. Since our main interest is in the transition from the hexatic to the distorted hexatic phases, it is sufficient to present only a typical crosssection of the phase diagram (described in the coordinates $a_{1}, a_{2}$ and $c_{1}$ ) in the region where the isotropic liquid is already unstable, $a_{1}<0$. In this cross-section with coordinates $\left(a_{2}, c_{2}\right)$ the phase diagram has a cusplike form, Fig. 11.

The relevant stable solutions of Eq. 固are in order of increasing density:

1. Isotropic 2D liquid: $|\psi|=0 ;|p|=0$

2. 2D hexatic phase: $|\psi| \neq 0 ;|p|=0$

3. Three different distorted hexatic phases with the property: $|\psi| \neq 0 ;|p| \neq 0$

(A) One with: $\sin 6(\phi-\theta)=0$ and $\cos 6(\phi-\theta)=1$, thus $\phi=\theta+\frac{\pi}{6}(2 n+1)$. Obviously here the polar vectors are directed along the molecular bonds.

(B) One with: $\sin 6(\phi-\theta)=0$ and $\cos 6(\phi-\theta)=-1$, thus $\phi=\theta+\frac{\pi n}{6}$. Here the polar vectors make an angle $\frac{\pi}{6}$ with the bond directions.

(C) Besides these two there also exists a lowersymmetry phase with a general angle between the polar vectors and the bond directions depending on external fields, such as density, and varying from $0^{\circ}$ to $30^{\circ}$.

The point orientational symmetry in 2D planes of these phases is $C_{6 v}$ for the hexatic phase, $C_{s}$ for distorted hexatic phases (A) and (B) and $C_{1}$ for the distorted hexatic phase $(\mathrm{C})$.

In a typical case the transitions hexatic $\longrightarrow$ distorted hexatic are of the second order. The transition from the hexatic phase to the low symmetry distorted hexatic phase (C) can be either the succession of two indirect 2nd order transitions or one direct 1st order transition, see Fig. 1. The distorted hexatic phases A and B are isostructural and therefore at least in principle there exists a continuous way to go between them without any phase transition. This scenario implies that the hexatic order parameter $|\psi|$ decreases in these distorted hexatic phases until it vanishes, goes through zero and reemerges with a different orientation of the polar vector with respect to the local hexagonal axes. More formally this scenario implies that $\phi-\theta$ should go from $0^{\circ}$ to $30^{\circ}$, a path which can be continuous only if it crosses the point $|\psi|=0$, where $\theta$ is not defined. If we assume that the hexatic order is strong enough and vanishes at no point in the phase diagram, then the only way from $\theta+\frac{\pi}{6}(2 n+1)$ to $\theta+\frac{\pi n}{6}$ distorted hexatic is through two phase transitions.

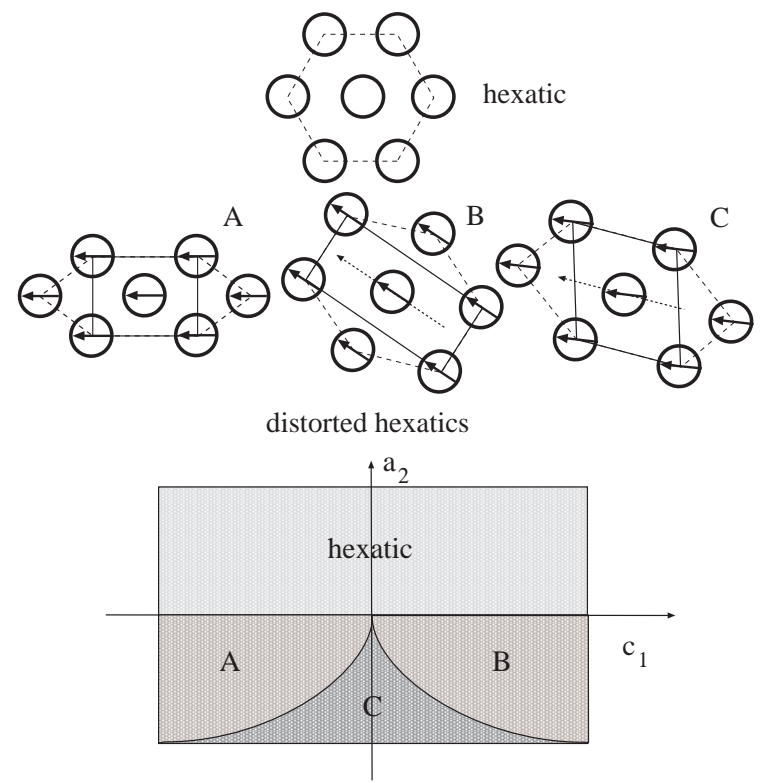

FIG. 1. The 2D section of the phase diagram in coordinates $a_{2}$ and $c_{1}$ for $a_{1}<0$, Eq. 1. The bold lines mark second order phase transition lines. The order of the transverse polarization vector can induce a transition into three types of distorted hexatic phases, where the polar ordering is coupled to the uniaxial distortions of the hexatic "unit cell" (there is no real unit cell in a hexatic). The hexatic order is indicated with dotted a hexagon. The directions of the transverse polarization vector in phase $(\mathrm{C})$ are in between $(\mathrm{A})$ and (B) as indicated by the arrows on the figure. The resulting elementary unit cells are shown by bold lines

The deformation of hexatic order (not shown in Fig. 1) of course follows the symmetry changes of the transverse polarization vector. It has to be uniaxial with one axis pointing in the direction of the transverse polarization vectors of the molecules. This can be seen very easily if we introduce a parameter quantifying the local distortion of the bond orientational (hexatic) field $\epsilon=|\epsilon| e^{i 2 \alpha}$, where $\epsilon$ is a combination of the plane strain tensor $\epsilon_{i j}$ components: $\epsilon=\epsilon_{x x}-\epsilon_{y y}+2 i \epsilon_{x y} ; \epsilon^{*}=\epsilon_{x x}-\epsilon_{y y}-2 i \epsilon_{x y}$.

The coupling terms that do exist are between the transverse polarization vector and the bond order distortion parameter and have the form $|p|^{2}|\epsilon| \cos 2(\phi-\alpha)$. This term induces the molecular bond distortion as a secondary (improper) order parameter, when $p$ is different 
from zero. In principle we should also add the coupling term between the distortion parameter and the hexatic order parameter that should be of the form $|\epsilon|^{3}|\psi| \cos 3(\alpha-\theta)$. This term shows that hexatic order in itself can not induce a distortion and a coupling of the distortion parameter to the polar order is crucial.

We now consider the case of larger densities where according to theoretical estimates [9] the preferred angle between transverse polarization vectors of neighboring molecules is non-zero. The transverse polarization order is thus inhomogeneous and we describe it with $\mathbf{p}(\boldsymbol{\rho})$, where $\boldsymbol{\rho}=(x, y)$. In this case the distorted hexatic phases can give way to lower symmetry phases characterized by a condensation of waves of the transverse polarization vector. The condensation of these modes invariably induces also long range positional order pushing the system from a distorted hexatic phase into a 2D crystalline phase with an orthorhombic unit cell.

If the wave condensation starts from the distorted hexatic phase A ( or B ), then, in general, for transverse polarization modes, there exist two qualitatively different choices of the wave vector $\mathbf{k}$ directions with respect to the symmetry plane of the distorted hexatic order Fig. 1. The direction of the mode can either find itself in the symmetry plane, in which case it can be described with a single wave vector, or it can point in a general direction, when it has to be described with the complete set of (two) directional wave vectors.

The first case can evidently describe only one dimensional modes. Indeed, here the direction of the mode along y-axis, characterised by $k_{y}$, is completely decoupled from the component along the x-axis. We thus obtain order parameter profiles which describe a onedimensional modulation of the transverse polarization orientation. Note that the polarization $\mathbf{p}$ is already ordered in the homogeneously distorted hexatic phases ; then the wave-like reorientation of $\mathbf{p}$ in the $(\mathrm{x}, \mathrm{y})$-plain is described by the wave of the pseudo-vector perpendicular to this plain and parallel to long molecular axes. The angle $\boldsymbol{\Omega}(\boldsymbol{\rho})$ of the reorientation of $\mathbf{p}$ with respect to its direction in the homogeneously distorted hexatic phase (i.e. A-phase) is given by the one-dimensional wave $\boldsymbol{\Omega}(\boldsymbol{\rho})=|\boldsymbol{\Omega}| \cos \left(k_{y} y+\beta\right)$, where $|\boldsymbol{\Omega}|$ is the module and $\beta$ is the phase of the corresponding order parameter (OP).

One of the possible realizations of the resulting ordered structure is presented as 1 in Fig. 2. Of course, the structure should depend on the relation between the wavelength of the modulation and the average distance between the molecules. This relation cannot be obtained in the framework of the phenomenological theory adopted in the present work and depends on the constants of the microscopic interaction between DNA molecules. For definiteness, we present here (structure 1 in Fig. 2) the limit case, where the wave vector of the modulation is taken to be $k_{y}=\frac{2 \pi}{b} \mathbf{e}_{y}$. Here $b$ is the long side of the orthorhombic "local unit cell" of the homogeneously distorted hexatic phase A (Fig. 1) ; $\mathbf{e}_{y}$ is a unit vector along the y-axis. Then the angles of the polarization $\mathbf{p}$ directions in adja- cent layers, taken with respect to the y-axis are $|\boldsymbol{\Omega}|$ and $-|\boldsymbol{\Omega}|$ respectively.

The only scalar invariant possible in the free energy descibing this transition is $|\boldsymbol{\Omega}|^{2}$ with the free energy itself assuming the form

$$
\mathcal{F}=d_{1}|\boldsymbol{\Omega}|^{2}+d_{2}|\boldsymbol{\Omega}|^{4}+\ldots
$$

Typically this free energy describes one 2 nd order phase transition at $d_{1}=0$. In the $\mathrm{x}$ direction, being the direction perpendicular to the direction of the condensed transverse polarization mode, the system remains liquid after the phase transition, with continuous translational symmetry.

In the second case the wave vector $\mathbf{k}$ is out of the symmetry plane of the $2 \mathrm{D}$ section of the system. The OP describing the transverse polarization reorientation mode is in general four dimensional. Mathematically the irreducible representation has two components but physically it has to have four components (if the waves are to be real). Therefore $\boldsymbol{\Omega}_{1}(\boldsymbol{\rho})=\left|\boldsymbol{\Omega}_{1}\right| e^{i \beta_{1}} e^{i \mathbf{k}_{1} \boldsymbol{\rho}} ; \boldsymbol{\Omega}_{2}(\boldsymbol{\rho})=$ $\left|\boldsymbol{\Omega}_{2}\right| e^{i \beta_{2}} e^{i \mathbf{k}_{2} \boldsymbol{\rho}} ; \boldsymbol{\Omega}_{3}(\boldsymbol{\rho})=\boldsymbol{\Omega}_{1}^{*}(\boldsymbol{\rho}) ;$ and $\boldsymbol{\Omega}_{4}(\boldsymbol{\rho})=\boldsymbol{\Omega}_{2}^{*}(\boldsymbol{\rho})$ , where $\mathbf{k}_{1}$ and $\mathbf{k}_{2}$ have the components $\mathbf{k}_{1}=\left(k_{x}, k_{y}\right)$ and $\mathbf{k}_{2}=\left(-k_{x}, k_{y}\right)$ along the symmetry axes of the "unit cell" of the homogeneously distorted hexatic phase ; angles $\left|\boldsymbol{\Omega}_{1}\right|$ and $\left|\boldsymbol{\Omega}_{2}\right|$ are two, in general independent wave amplitudes. The most general form of the resulting wave, which again has to be real, is $\boldsymbol{\Omega}(\boldsymbol{\rho})=\sum_{i} \boldsymbol{\Omega}_{i}=$ $\left(\left|\boldsymbol{\Omega}_{1}\right| \cos \left(\mathbf{k}_{1} \boldsymbol{\rho}+\beta_{1}\right)+\left|\boldsymbol{\Omega}_{2}\right| \cos \left(\mathbf{k}_{2} \boldsymbol{\rho}+\beta_{2}\right)\right)$, with $\beta_{1}$ and $\beta_{2}$ being the initial phases of the OP components that can be made zero by an appropriate choice of the origin.

The free energy of the transverse polarization reorientation described by this OP depends only on two independent invariants : $I_{1}=\left|\Omega_{1}\right|^{2}+\left|\Omega_{2}\right|^{2}$ and $I_{2}=\left|\Omega_{1}\right|^{2}\left|\Omega_{2}\right|^{2}$. The corresponding free energy thus assumes the form

$$
\mathcal{F}=d_{1} I_{1}+d_{2} I_{1}^{2}+f_{1} I_{2}+\ldots
$$

Apart from the distorted hexatic phases $\mathrm{A}, \mathrm{B}$ or $\mathrm{C}$ with homogeneous transverse polarization order $\left|\boldsymbol{\Omega}_{1}\right|=$ $0 ;\left|\boldsymbol{\Omega}_{2}\right|=0$ already dealt with above, the minimization of this free energy gives three different stable states:

$2 \mathrm{a}\left|\boldsymbol{\Omega}_{1}\right| \neq 0 ;\left|\boldsymbol{\Omega}_{2}\right|=0$ or another domain where the roles of the fields $\left|\boldsymbol{\Omega}_{1}\right|$ and $\left|\boldsymbol{\Omega}_{2}\right|$ are reversed,

2b $\left|\boldsymbol{\Omega}_{1}\right|=\left|\boldsymbol{\Omega}_{2}\right|$, with either $\boldsymbol{\Omega}_{1}=\boldsymbol{\Omega}_{2}$ or $\boldsymbol{\Omega}_{1}=-\boldsymbol{\Omega}_{2}$,

$2 \mathrm{c}$ or the most general form $0 \neq\left|\boldsymbol{\Omega}_{1}\right| \neq\left|\boldsymbol{\Omega}_{2}\right| \neq 0$.

In what follows we shall limit ourselves to the distorted hexatic A and the phases with nonhomogeneous transverse polarization order that are derived from it, since the positional order in the crystalline phase of DNA strongly favors this type of distortion [8].

The structure 2a, see Fig. 2, has discrete translational order only in one dimension with (as opposed to structure 1) the wave vector making some general angle with the symmetry plane of the uniformly distorted hexatic phase. The transverse polarization reorientation mode can be 
described with a simple form $\boldsymbol{\Omega}(\boldsymbol{\rho})=\left|\boldsymbol{\Omega}_{1}\right| \cos \left(\mathbf{k}_{1} \boldsymbol{\rho}\right)$. The direction perpendicular to $\mathbf{k}_{1}$ remains liquid. Again for definiteness, we present the limit case where $\mathbf{k}_{1}$ is taken to be $\mathbf{k}_{1}=\frac{\pi}{a} \mathbf{e}_{x}+\frac{\pi}{b} \mathbf{e}_{y}, a$ and $b$ being the parameters of the "local unit cell" of the phase A.

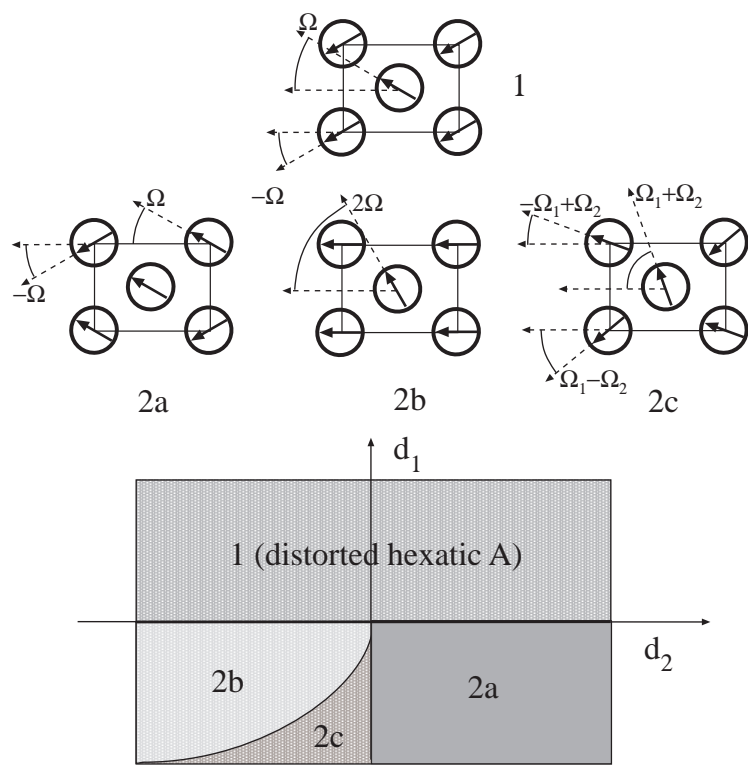

FIG. 2. The $2 \mathrm{D}$ section of the phase diagram in coordinates $d_{1}$ and $d_{2}$ for $f_{1}>0$, Eq. 3. Distorted hexatic A, see Fig. 1. can give way to crystalline low symmetry phases with condensed transverse polarization modes. In (1) the transverse polarization reorientation mode condenses only in one direction coinciding with one of the distorted hexatic "unit cell" vectors. In phases (2) the angle $\boldsymbol{\Omega}$ of the polarization reorientation is a sum of two modes with wave vectors out of the symmetry plane of the $2 \mathrm{D}$ section, where the wave amplitudes $\left|\boldsymbol{\Omega}_{i}\right|$ are in general independent.

The structure 2b, see Fig. 2, is represented by the sum of two transverse polarization modes in the directions of $\mathbf{k}_{1}$ and $\mathbf{k}_{2}$ with equal amplitudes. The polarization reorientation mode thus assumes the form $\boldsymbol{\Omega}(\boldsymbol{\rho})=$ $\left|\boldsymbol{\Omega}_{1}\right|\left(\cos \left(\mathbf{k}_{1} \boldsymbol{\rho}\right)+\cos \left(\mathbf{k}_{2} \boldsymbol{\rho}\right)\right)$. Respecting the symmetry of the wave vectors we present the limit case where $\mathbf{k}_{1}$ and $\mathbf{k}_{2}$ are chosen in the form : $\mathbf{k}_{1}=\frac{\pi}{a} \mathbf{e}_{x}+\frac{\pi}{b} \mathbf{e}_{y}$ and $\mathbf{k}_{2}=-\frac{\pi}{a} \mathbf{e}_{x}+\frac{\pi}{b} \mathbf{e}_{y}$. Note that the structures 1, 2a and $2 \mathrm{~b}$ can be described using only one angular amplitude (see Fig. 2).

The structure 2c, see Fig. 2, presents the most general structure of the ordered phase with two different directions of the transverse polarization modes, $\mathbf{k}_{1}$ and $\mathbf{k}_{2}$ but also with different amplitudes of the waves $\left|\boldsymbol{\Omega}_{1}\right| \neq\left|\boldsymbol{\Omega}_{2}\right|$. To present the limit structure we choose the wave vectors $\mathbf{k}_{1}$ and $\mathbf{k}_{2}$ in the same form as for the previous phase. The symmetry is broken with respect to the phases $2 \mathrm{a}$ and $2 \mathrm{~b}$ : the directions of the transverse polarizations of the molecules now make three different angles with respect to the y-axis $\left|\boldsymbol{\Omega}_{1}\right|+\left|\boldsymbol{\Omega}_{2}\right|,\left|\boldsymbol{\Omega}_{1}\right|-\left|\boldsymbol{\Omega}_{2}\right|$ and $-\left|\boldsymbol{\Omega}_{1}\right|+\left|\boldsymbol{\Omega}_{2}\right|$.

The phase diagram corresponding to the free energy
Eq. 3 has the form as presented on Fig. 2. All the transitions between the distorted hexatic phase and modulated phases, as well as between the modulated phases are of second order.

The existence of angular interactions among stiff, nematically ordered, polymer molecules such as DNA, that depend on the orientation of their transverse polarization vectors can introduce important modifications into the phase diagram at intermediate and high densities. If the equilibrium transverse polarization order is homogeneous (intermediate densities), corresponding to aligned transverse polarization vectors on neighboring molecules, than the hexatic phase becomes distorted with one axis of the local "unit cell" pointing in the direction of the transverse polarization order. This distortion, that should grow as one approaches the hexatic - crystal transition, could show up through a broader positional peak in Xray scattering, corresponding to domains of different distorted hexatic directions, giving a very satisfactory explanation for observations in DNA [8].

If on the other hand, the equilibrium transverse polarization order is inhomogeneous (higher densities), corresponding to non-zero angles between neighboring transverse polarization vectors, the corresponding lattice becomes crystalline with long range positional order either in one or both directions and with a deformed hexagonal unit cell. Thus instead of having six equivalent nearest neighbors, the distorted hexagonal unit cell can give way to four nearest neighbors at a non-zero angle (energetically more favourable) between $\boldsymbol{\Omega} \mathrm{s}$, whereas the more distant molecules can have the same direction of $\boldsymbol{\Omega}$ (energetically less favourable). This case too could be associated directly with observations in crystallised DNA arrays 《A. We are thus able to provide a consistent interpretation for DNA phase behavior in a range of densities between the crystalline and the cholesteric phases.

[1] R. Podgornik, H.H. Strey and V.A. Parsegian Curr. Opin. Coll. \& Interf. Sci. 3 (1998) 534.

[2] D.R. Nelson, Nature 375 (1995) 356.

[3] R. Podgornik, H.H. Strey and V.A. Parsegian, in Gene Therapy: Therapeutic Mechanisms and Strategies, N. Smyth Templeton and Lasic D.D. (Eds.), Marcel Dekker, New York (2000) 209.

[4] R. Langridge et al., J. Mol. Biol. 2 (1960) 19.

[5] H. Grimm and A. Rupprecht, Physica B 174 (1991) 291.

[6] G. Toulouse, Commun. Phys. 2, 115 (1977). E. B. Sirota, Langmuir 13, 3849 (1997).

[7] R. Podgornik et al., Proc. Natl. Acad. Sci. 93 (1996) 4261.

[8] H.H. Strey et al. , Phys. Rev. Lett. 84 (2000) 3105.

[9] E. Allahyarov and H. Löwen, Phys. Rev. E 62 (2000) 5542. A.A. Kornyshev and S. Leikin, J. Chem. Phys. 107 (1997) 3656 . 